\title{
Flare magnetic reconnection and relativistic particles in the 2003 October 28 event
}

\author{
C. $\mathrm{Li}^{1,2}$, Y. H. Tang ${ }^{1}$, Y. Dai ${ }^{1}$, C. Fang ${ }^{1}$, and J.-C. Vial ${ }^{2}$ \\ 1 Department of Astronomy, Nanjing University, Nanjing 210093, PR China \\ e-mail: chuan.li@ias.u-psud.fr; lic@nju.edu.cn \\ 2 Institut d'Astrophysique Spatiale, Bâtiment 121, Université Paris-sud 11 and CNRS, Orsay 91405, France
}

Received 19 December 2006 / Accepted 5 June 2007

\begin{abstract}
An X17.2 solar flare occurred on 2003 October 28, accompanied by multi-wavelength emissions and a high flux of relativistic particles observed at $1 \mathrm{AU}$. We present the analytic results of the TRACE, SOHO, RHESSI, ACE, GOES, hard X-ray (INTEGRAL satellite), radio (Ondeřejov radio telescope), and neutron monitor data. It is found that the inferred magnetic reconnection electric field correlates well with the hard X-ray, gamma-ray, and neutron emission at the Sun. Thus the flare's magnetic reconnection probably makes a crucial contribution to the prompt relativistic particles, which could be detected at 1 AU. Since the neutrons were emitted a few minutes before the injection of protons and electrons, we propose a magnetic-field evolution configuration to explain this delay. We do not exclude the effect of CME-driven shock, which probably plays an important role in the delayed gradual phase of solar energetic particles.
\end{abstract}

Key words. acceleration of particles - Sun: magnetic fields - Sun: flares

\section{Introduction}

When a high flux of relativistic solar nucleons strikes Earth's atmosphere, the straightforward neutrons and/or the nuclear byproducts can result in a "ground level enhancement" (GLE). However, the exact acceleration sources of the relativistic particles remain enigmatic. The main controversy focuses on acceleration occurring at shocks driven by coronal mass ejections (CMEs) or in the active region producing flares (Kahler 1994; Reames 1999, 2002; Cane et al. 2002; Kallenrode 2003). The acceleration during the process of the coronal magnetic field reconfiguration at heights between $0.1 R_{\mathrm{S}}$ and $1 R_{\mathrm{S}}$ above the photosphere, neither in the flare active region nor at the bow shock of the CME, is also suggested by Klein et al. (2001).

Magnetic reconnection in the active region is a candidate for a large energy release at the Sun. When a coronal flux rope loses equilibrium and travels upwards, below which an extensive reconnection current sheet (RCS) forms, the reconnection in this RCS releases most of the magnetic energy stored in the configuration (Forbes \& Priest 1995; Lin \& Forbes 2000). Charged particles can be effectively accelerated by the induced reconnection electric field in the RCS (Martens \& Young 1990; Livenenko \& Somov 1995).

In this paper, we evaluate the induced reconnection electric field of the X17.2 two-ribbon flare that occurred on 2003 October 28 and compare it with multi-wavelength and particle observations. Our results reveal that the reconnection electric field probably plays an important role in accelerating relativistic nucleons and make a crucial contribution to the prompt impulsive phase of solar energetic particles (SEPs).

\section{Observations and data analysis}

\subsection{Flare magnetic reconnection}

The GOES X17.2 two-ribbon flare located in the NOAA active region 10486 (S16E08) began at 09:40 UT, reached its peak at 11:10 UT and ended around 11:25 UT. Figure 1 shows the RHESSI hard X-ray sources superimposed on the TRACE $195 \AA$ image. Red contour lines indicate $12-25 \mathrm{keV}$ thermal bremsstrahlung sources integrated from 11:10:40 to 11:20:40 UT after the flare's peak, and blue ones indicate 200-300 keV non-thermal sources integrated from 11:06:40 to 11:20:40 UT during the flare's peak and decay phase. It can be found that the three non-thermal sources are located just on a flare-post-loop structure, and the thermal ones straddle the arcade of the loops.

It is now well-recognized that the flare ribbon's expansion is the chromospheric signature of the progressive magnetic reconnection in the corona in which new field lines reconnect at higher and higher altitudes. The separation motion of flare ribbons that sweep through the magnetic field lines corresponds to the rate of magnetic reconnection in the corona, where the reconnection current sheet (RCS) is generated. We measured the magnetic reconnection rate in the form of a reconnection electric field, which can be given by $E_{\text {rec }}=V B$ (Forbes \& Lin 2000; Qiu et al. 2002), where $V$ is the separation velocity of flare ribbons and $B$ the magnetic field that the ribbons sweep through.

Since the flare occurred near disk center, $B$ can be approximately taken as the longitudinal component of the magnetic field obtained from the SOHO/MDI magnetogram at 11:11:03 UT. We use the high-cadence $(\sim 1 \mathrm{~min}) 195 \AA$ TRACE observations covering the time interval of 10:47 UT-11:26 UT to measure 


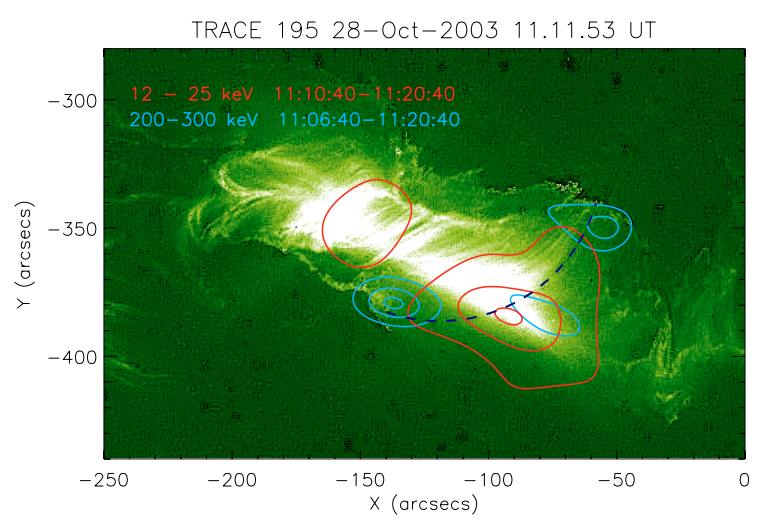

Fig. 1. RHESSI hard X-ray sources (Rotating modulation collimators 4-8, with MEM-Sato image reconstruction algorithm) overlaid on the TRACE $195 \AA$ image at 11:11:53 UT. Sky-blue contour lines indicate $200-300 \mathrm{keV}$ hard X-ray sources integrated from 11:06:40 to 11:20:40 UT, and red 12-25 keV integrated from 11:10:40 to 11:20:40 UT. Dashed blue line indicates the flare-post-loop structure.

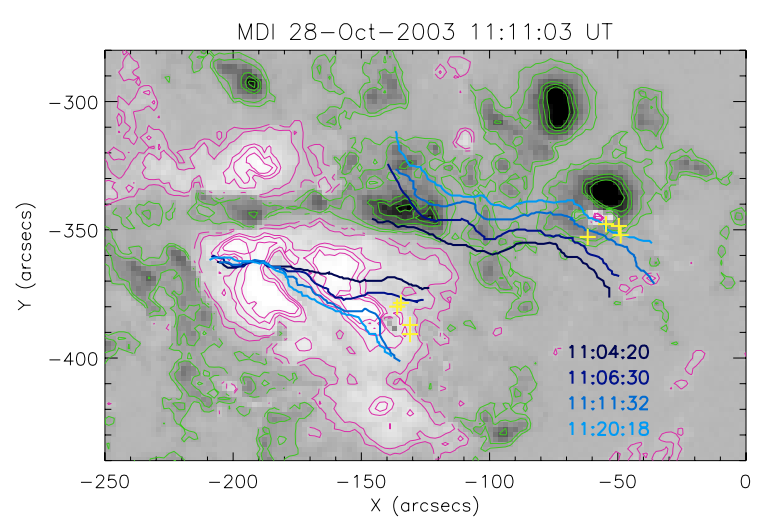

Fig. 2. Positions of the TRACE EUV ribbons and RHESSI hard $\mathrm{X}$-ray footpoints overlaid on the magnetogram. Blue lines indicate the EUV ribbons at different times, and yellow + signs indicate 200-300 keV footpoints for successive integrated time intervals of 100 , 130, 180, and $240 \mathrm{~s}$ beginning at 11:06:20 UT. Red contours lines indicate positive longitudinal magnetic field, and green negative.

the flare ribbon's separation. The positions of the flare EUV ribbons and hard X-ray footpoints were overlaid on the magnetogram (see Fig. 2, detailed processing methods are discussed in $\mathrm{Li}$ et al. 2006). There is no RHESSI data before 11:06:20 UT, so we reconstruct successive footpoints of 200-300 keV sources between 11:06:20 and 11:10:20 UT (Fig. 2). We can find that the general moving directions of the two hard X-ray footpoints are toward the south and west and follow the separation of the flare ribbons. This can be evidence in support of the model and method for calculating the reconnection rate.

From the MDI magnetogram and the measurement of the flare ribbon's separation, we get $B$ and $V$. Then the induced reconnection electric field can be evaluated. In Fig. 3, the inferred reconnection electric field is shown in comparison with multiwave observations and time profiles of solar neutrons. It is found that the reconnection electric field has, generally speaking, a good temporal correlation with hard X-ray and microwave emission, especially with the neutron capture line and the increase profile of neutron monitor data. As we know, accelerated protons and nuclei can produce high-energy neutrons through nuclear reactions from inelastic collisions in the solar atmosphere, and this process also generates high energy gamma rays, so the good temporal correlation indicates a physical link between magnetic

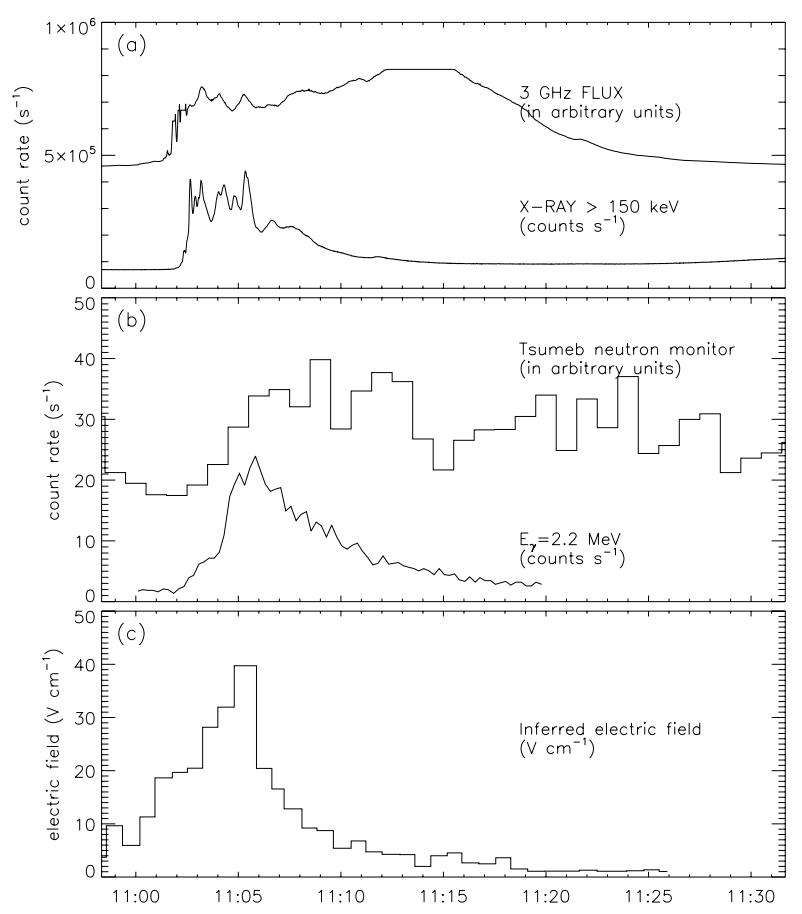

Fig. 3. a) Hard X-ray emission (INTEGRAL satellite) and $3 \mathrm{GHz}$ radio emission (Ondeřejov radio telescope). b) Neutron capture line at $2.223 \mathrm{MeV}$ (Kiener et al. 2006, Fig. 2) and Tsumbe neutron monitor one-minute-averaged count rate. c) Reconnection electric field $E_{\mathrm{rec}}$ inferred from the X17.2 two-ribbon flare.

reconnection and energy release in flares, also suggests that the reconnection electric field $E_{\text {rec }}$ plays an important role in accelerating nonthermal charged particles, both electrons and ions.

One may suggest different spatial accelerations between electrons and ions in solar flares. However, INTEGRAL/SPI observations (Kiener et al. 2006, Figs. 1 and 2) show that the electron-associated hard X-ray emission and the ion-associated prompt $\mathrm{C}$ and $\mathrm{O}$ de-excitation lines ( 4.4 and $6.6 \mathrm{MeV}$ ) have very similar profiles. Comparing the beginning of the bremsstrahlung emission and the edge of the neutron capture line increase, a typical neutron thermalization time $\sim 100 \mathrm{~s}$ has been observed (Gros et al. 2004, Fig. 6). Taking this time delay into account, Hurford et al. (2006) find that the two $2.223 \mathrm{MeV}$ gamma-ray sources have about the same separation as the two corresponding 200-300 keV electron bremsstrahlung sources with a displacement of 14 and $17 \pm 5$ arcsec. Thus the electrons and ions appear to have the same acceleration source. A very simple explanation of the displacement is that electrons and ions accelerated in the RCS will travel along different magnetic fields due to gradient or curvature drift; in other words, electrons are much more magnetic-controlled than ions.

In the RCS, charged particles can be accelerated by the induced reconnection electric field. For this event, given the maximum $E_{\mathrm{rec}} \sim 40.0 \mathrm{~V} / \mathrm{cm}$, an acceleration length $l_{\text {acc }} \sim 2.5 \times$ $10^{7} \mathrm{~cm}$ is needed to accelerate the protons to $\mathrm{GeV}$ energy. The ratio of the acceleration length to the whole flare ribbon's length is $\sim 4.0 \times 10^{-3}$, hence protons are not accelerated in a single beam running the full length of the RCS. This avoids the contradiction that the electric current associated with the accelerated particles would be so strong that the induced magnetic field would greatly exceed typical coronal values. 


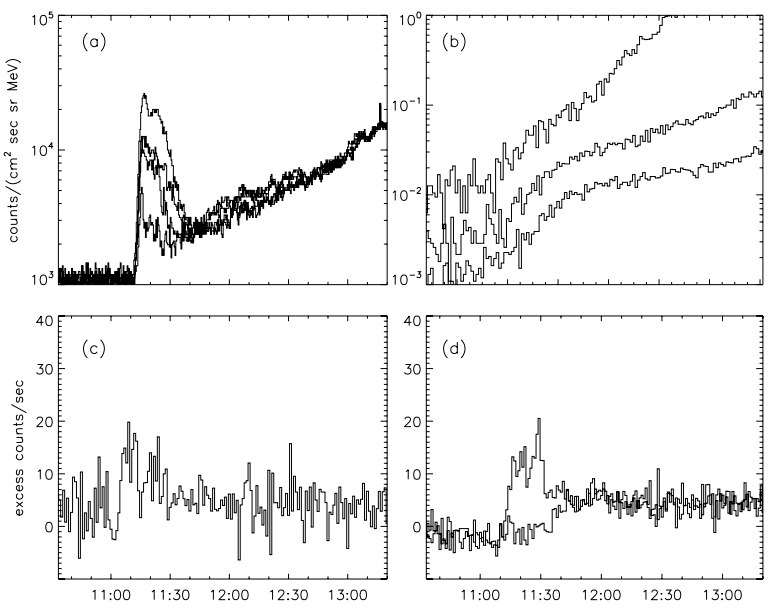

Fig. 4. a) The intensity of ACE/EPAM $103-175 \mathrm{keV}$ electron channel, all four sectors are plotted. b) GOES-10 $\mathrm{P}_{5}, \mathrm{P}_{6}$, and $\mathrm{P}_{7}$ channel data are 1-min-averaged and cover the energy ranges 40-80, 80-165, and 165-500 MeV, respectively. c) Tsumbe neutron monitor 1-minaveraged data. d) Apatity and Moscow neutron monitor 1-min-averaged data.

\subsection{Solar energetic particles}

During this large solar flare, the near-equatorial neutron monitor in Tsumeb, with high cutoff rigidity of $9.12 \mathrm{GV}$, first observed an enhancement above background before the arrival of solar protons, which was attributed to direct solar neutrons (Plainaki et al. 2004; Struminsky 2005). Then a few minutes later, several other stations, such as Moscow and Apatity neutron monitors, detected excess count rate of solar cosmic rays (SCRs). A high flux of protons and electrons were also recorded by GOES and ACE several minutes later. Figure 4 shows increase profiles of SEPs and SCRs recorded by satellites and ground-based neutron monitors at $1 \mathrm{AU}$.

The neutrons emitted at the Sun follow a straight line path from the emission point to the Earth. According to Tsumeb neutron monitor data (Fig. 4, panel c), we estimate the neutron's emission time is 11:05 UT $\pm 1 \mathrm{~min}$.

Assuming that protons and electrons travel along the interplanetary magnetic field (IMF) lines at a speed of $v$ with no scattering, in order to compare with multi-wavelength observations, we estimate the solar release time by subtracting $\Delta t$ from observed time at $1 \mathrm{AU}$, where $\Delta t=1.1 \mathrm{AU} / v-8.3 \mathrm{~min}$, and 1.1 AU corresponds to the length of IMF lines when the solar wind is about $700 \mathrm{~km} \mathrm{~s}^{-1}$ (ACE/SWEPAM) for this event. From ACE/EPAM, GOES-10, and neutron monitors data (Fig. 4, panels $\mathrm{a}, \mathrm{b}$, and d), the evaluated proton's and electron's injection time is $11: 12 \mathrm{UT} \pm 1 \mathrm{~min}$. It is close to the result of Bieber et al. (2005), who infer the relativistic protons were injected at $\sim 11: 11 \mathrm{UT}$.

This GLE event was also accompanied by a fast halo CME. Using the data from the LASCO CME catalog, extrapolating the halo $\mathrm{CME}$ to the solar disk center (quadratic fit), we estimate the upper limit of the CME liftoff is $\sim 11: 07 \mathrm{UT}$, which is later than the neutron's emission of $\sim 11: 05$ UT. It indicates that the CME-driven shock plays a minor role in producing relativistic neutrons, which probably come from the byproducts of charged particles accelerated in the active region. Hurford et al. (2006) also suggest that the gamma-ray producing ions appear to be accelerated by the flare process and not by a widespread shock driven by a fast CME.

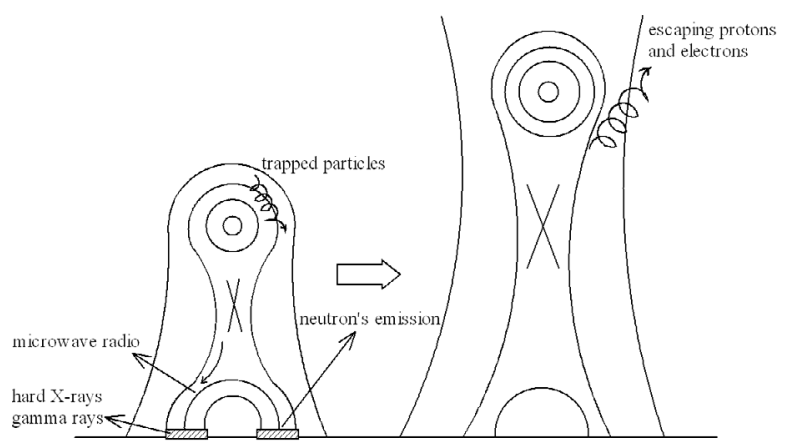

Fig. 5. Sketch of the proposed model for the magnetic field configuration associated with the flare and plasmoid ejection. The subsequent neutron emission, and the proton and electron injection are shown in the two phases from left to right.

\subsection{Magnetic field configuration}

This event shows another interesting aspect: if relativistic charged particles (protons and electrons) and their byproduct neutrons observed at $1 \mathrm{AU}$ are produced almost at the same time in the active region as we suggest, why is the neutron emission (11:05 UT $\pm 1 \mathrm{~min})$ a few minutes before the proton and electron injection $(11: 12 \mathrm{UT} \pm 1 \mathrm{~min})$. To explain this phenomena, we propose the magnetic-field evolution configuration shown in Fig. 5. This scenario is based on the model of flares with flux rope ejection (Ohyama \& Shibata 1998; Lin \& Forbes 2000).

First due to some instability, the coronal flux rope loses equilibrium and travels upwards, below which the RCS forms. Charged particles accelerated in the RCS travel downwards along magnetic field lines and generate microwave emission due to synchrotron, hard X-ray emission due to bremsstrahlung, gamma-ray emission due to nuclear reaction, and high energy neutrons in the process of gamma-ray generation. The produced neutrons follow a straight line path from the emission point to the Earth undisturbed by magnetic fields and are detected by the neutron monitors on the ground. However, the charged particles are probably trapped by the closed magnetic fields and cannot escape from the active region.

Then a few minutes later (around 11:12 UT), magnetic fields reconnect at a higher altitude, corresponding to the flare's expansion in the chromosphere, and the flux rope also travels to a much higher site and is ejected. During this process, open field lines should be produced (Shibata 2006), and charged particles (protons and electrons) accelerated in the RCS could escape along open field lines into the interplanetary space and be detected at $1 \mathrm{AU}$.

In fact, during this proposed evolution of the magnetic field configuration, a large-scale coronal disturbance was observed (Dai et al. 2005). From the running difference EIT $195 \AA$ images (shown in Fig. 6), it is found that around 11:12 UT, a large amount of coronal material was ejected, corresponding to the process from the coronal brightening to dimming. This process may open quite a lot of magnetic field lines in the low corona, facilitating the flare accelerated particles along these open field lines into the interplanetary space.

\section{Discussion}

The X17.2 flare that occurred on 2003 October 28 in the active region NOAA 10486 (S16E08) is nominally not well-connected with the Earth, and this is an argument against flare acceleration of solar protons. However, because of the high-speed solar 

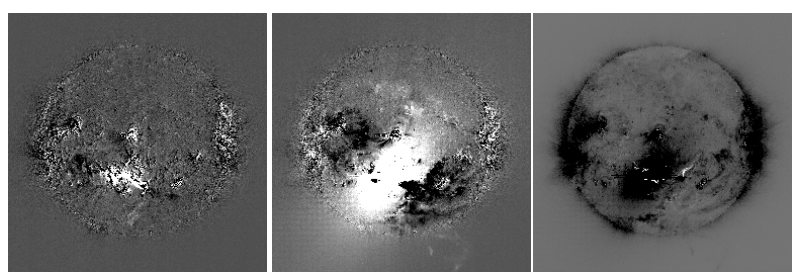

Fig. 6. The coronal disturbance on 2003 October 28. The three images show EIT $195 \AA$ at 11:00, 11:12, and 11:24 UT with a pre-event image subtracted from them.

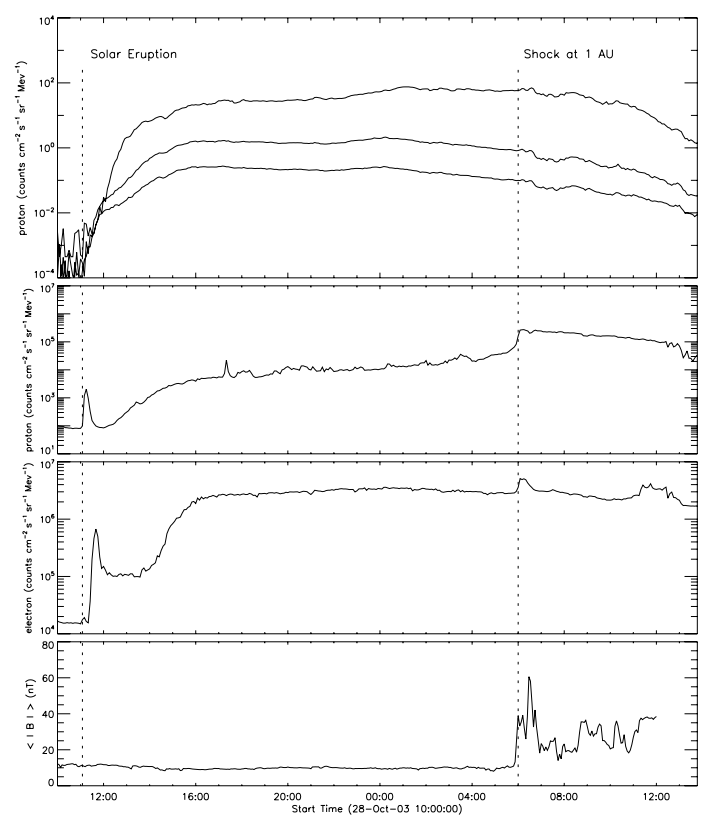

Fig. 7. Temporal profiles of the energetic protons, electrons intensity, and magnetic field near $1 \mathrm{AU}$ of the 2003 October 28 event. In the upper panel, the GOES-10 $\mathrm{P}_{5}, \mathrm{P}_{6}$, and $\mathrm{P}_{7}$ channel data cover the energy ranges $40-80,80-165$, and $165-500 \mathrm{MeV}$, respectively. Lower energetic protons $(0.31-0.58 \mathrm{MeV})$ and electrons $(0.038-0.053 \mathrm{MeV})$ from $\mathrm{ACE} / \mathrm{EPAM}$ are shown in the middle two panels and magnetic field from $\mathrm{ACE} / \mathrm{MAG}$ at bottom.

wind $\left(\sim 700 \mathrm{~km} \mathrm{~s}^{-1}\right)$, the evaluated footpoint of the IMF is located at $\sim \mathrm{W} 30$, not very far from the flare site. Moreover the large-scale solar-surface disturbance (shown in Fig. 6) could offer a particle transport path from the flare site to the wellconnected region and may correspond to a field-opening process. A loop-like IMF line formed by a preceding CME on 2003 October 26 connecting the Earth with the flare was also proposed by Bieber et al. (2005) and Miroshnichenko et al. (2005).

This GLE event displayed an initial impulsive increase corresponding to the solar eruption and was followed by a gradual component until the CME driven shock arrived at the Earth, as Fig. 7 shows. It appears that there are two populations of SEPs: the prompt one causes an impulse-like increase and the delayed one has a slow intensity rise, especially for the lower energetic particles, as shown in the middle two panels. The prompt solar energetic particles could be well-explained by the acceleration in the flare active region; however, the following delayed ones cannot stem from only the active region because of their long-duration injection. Some of the particles accelerated in the active region would be trapped or reaccelerated by the coronal CME-driven shock, and the interplanetary CME-driven shock could also add some particles to the delayed injection.

From Fig. 7, it is also found that, when the CME-driven shock reached $1 \mathrm{AU}$ at $\sim 6: 00$ UT Oct. 29, the lower energetic particles (ACE/EPAM 0.31-0.58 MeV protons and $0.038-0.053 \mathrm{MeV}$ electrons) showed obvious increase. However, the relatively much higher energetic particles (GOES several tens of $\mathrm{MeV}$ protons) show nearly no increase. This indicates that CME-driven shocks play a minor role in accelerating higher energetic, especially the relativistic particles.

The GLE event of 2003 October 28 presents many unusual features all of which we do not explain. From the analysis of multi-wavelength observations and energetic particle data, we propose that the flare magnetic reconnection, especially the induced electric field, makes a crucial contribution to the prompt relativistic particles. On the other hand, the CME-driven shock probably plays an important role in the delayed gradual injection of SEPs, especially in the lower energetic ones.

Acknowledgements. We are very grateful to the referee Dr. E. Kontar, whose constructive comments have greatly improved this paper. We thank the TRACE, SOHO, RHESSI, and ACE teams for providing the observational data. The INTEGRAL satellite and Ondeřejov radio telescope data were kindly provided by Dr. M. Karlický. We thank the Polar Geophysical Institute (Russia) and Unit of Space Physics (South Africa) for providing the neutron monitor data. This work was supported by NSFC key projects Nos. 10333040, 10221001, and NKBRSF of China G2006CB806300.

\section{References}

Bieber, J. W., Clem, J., Evenson, P., et al. 2005, Geophys. Res. Lett., 32, 3

Cane, H. V., Erickson, W. C., \& Prestage, N. P. 2002, J. Geophys. Res., 107, 1315

Dai, Y., Tang, Y. H., \& Qiu, K. P. 2005, Prompt solar energetic particles with large-scale cross-disk coronal disturbance, Proc. IAU Symp., 226, 374

Forbes, T. G., \& Priest, E. R. 1995, ApJ, 446, 377

Forbes, T. G., \& Lin, J. 2000, J. Atmos. Sol-Terr. Phys., 62, 1499

Gros, M., Tatischeff, V., Kiener, J., et al. 2004, Proceedings of the 5th INTEGRAL Workshop, 16-20 February 2004, Munich, Germany, 669

Hurford, G. J., Krucker, S., Lin, R. P., et al. 2006, ApJ, 644, 93

Kahler, S. W. 1994, ApJ, 428, 837

Kallenrode, S. W. 2003, J. Phys. G: Nucl. Part. Phys., 29, 965

Kiener, J., Gros, M., Tatischeff, V., \& Weidenspointner, G. 2006, A\&A, 445, 725 Klein, K.-L., Trottet, G., Lantos, P., \& Delaboudinière, J.-P. 2001, A\&A, 373, 1073

Li, C., Tang, Y. H., Dai, Y., Zong, W. G., \& Fang, C. 2007, A\&A, 461, 1115

Lin, J., \& Forbes, T. G. 2000, J. Geophys. Res., 105, 2375

Litvinenko, Y. E., \& Somov, B. V. 1995, Sol. Phys., 158, 317

Martens, P. C. H., \& Young, A. 1990, ApJS, 73, 333

Miroshnichenko, L. I., Klein, K.-L., Trottet, G., Lantos, P., et al. 2005, J. Geophys. Res. [doi : 10.1029/2004]A010936]

Ohyama, M., \& Shibata, K. 1998, ApJ, 499, 934

Plainaki, C., Belov, A., Eroshenko, E., et al. 2004, Unexpectable burst of solar activity recorded by neutron monitors during October-November 2003, presented at 35th COSPAR Scientific Assembly, Comm. on Space Res., Paris, June

Qiu, J., Lee, J., Gary, D. E., \& Wang, H. M. 2002, ApJ, 565, 1335

Reames, D. V. 1999, Space Sci. Rev., 90, 413

Reames, D. V. 2002, ApJ, 571, 63

Shibata, K. 2006, Solar eruption and magnetic reconnection, presented at Western Pacific Geophysics Meeting, Beijing, China, July

Struminsky, A. B. 2005, On possiblility of prolonged two step production of high energy neutrons during solar flare on 28 October 2003, Proc. 29th Int. Cosmic Ray Conf. Pune, India, August 